\title{
Within-category discrimination of musical chords: Perceptual magnet or anchor?
}

\author{
BARBARA E. ACKER, RICHARD E. PASTORE, and MICHAEL D. HALL \\ State University of New York, Binghamton, New York
}

\begin{abstract}
Recent speech research has begun to evaluate the internal structure of categories. In one such study, Kuhl (1991) found that discrimination was poorer for vowel stimuli that were more representative of the category (prototype, or P, set) than it was for less representative stimuli (nonprototype, or NP, set). This finding was interpreted as indicating that a category prototype may function as a "perceptual magnet," effectively decreasing perceptual distance, and thus discriminability, between stimuli. The present study examines the function of prototypes in a musical category-another natural, but nonspeech category. Paralleling the Kuhl study, representative (P) and less representative (NP) sets of major triad stimuli were constructed, based on equal temperament. Musically experienced subjects rated the stimuli in each set for goodness as a major triad, with the highest rated stimulus serving as a prototype standard for a subsequent discrimination task. Results from the discrimination task demonstrated better performance in the P context than in the NP context. The current nonspeech results indicate that a prototype functions as an anchor rather than a magnet. In addition to providing a natural, nonspeech standard for comparison with speech findings, the results provide some important insights into the nature of musical categories.
\end{abstract}

Although it has long been conjectured that speech categorization may be based on the use of prototypes or exemplars, most speech perception research has tended to focus on the location of category boundaries. This focus on category boundaries is probably a carryover from the notions of categorical perception which posited absolute recoding of perception in terms of discrete phonetic categories, and which dismissed any within-category perceptual variation as being due to stimulus artifacts (StuddertKennedy, Liberman, Harris, \& Cooper, 1970). As a result, little attention has been given to differences in perceptual quality or goodness, which, in theory, should exist within categories that are based on the use of prototypes or exemplars. In contrast to this long tradition of categorization studies based on labeling tasks, some recent research has begun to examine the internal structure of speech categories (e.g., Kuhl, 1991; Li \& Pastore, 1992; Samuel, 1982; Volatis \& Miller, 1992). In general, these studies have found category membership to be qualitatively graded, with Kuhl (1991) providing evidence that quality of membership in a vowel category is reflected in a distinct pattern of discriminability between stimuli. Patterns of qualitative grading and related patterns of discrimination

This research was supported by Grants F496209310033 and F49069310327 from the Air Force Office of Scientific Research to the second author. The opinions expressed are those of the authors and do not necessarily represent those of the granting agency. We would like to thank Cynthia Connine for several helpful suggestions concerning the discrimination models. Correspondence should be addressed to $B$. Acker, Psychoacoustics and Auditory Cognition Laboratory, Department of Psychology, State University of New York, Binghamton, NY 13902-6000 (e-mail: bb31074@bingvmb.cc.binghamton.edu). within categories may be indicative of the nature of general category structure and could occur in both speech and nonspeech categories. In the present paper, we evaluate the qualitative distribution of category membership and the pattern of discriminability for a musical category, which thus represents another natural, ${ }^{1}$ but nonspeech, category.

\section{The "Perceptual Magnet"}

In examining the internal structure of an $/ \mathrm{i} /$ vowel category with stimuli varying in F1 and F2 (F3, F4, and F5 were constant), Kuhl (1991) demonstrated a "perceptual magnet" effect. Kuhl constructed prototype (P) and nonprototype (NP) sets of /i/ stimuli, with the $\mathrm{P}$ stimuli being more representative of the /i/ category than the NP stimuli. Whereas Figure 1 depicts stimuli from the current study, it provides a close approximation to the Kuhl stimulus set by substituting F1 for E (abscissa) and F2 for G (ordinate), and having the stimuli change in steps of $30 \mathrm{Mels}$ instead of $2 \mathrm{~Hz}$. The dotted diagonal line generally separates the $P$ (upper left) context from the NP (lower right) context, except that the stimuli labeled "P," "N," and "o" are common to both contexts. Subjects rated the goodness of stimuli in each context. The theoretical prototype (analogous to the stimulus labeled "P" in Figure 1) was based on the average $F 1$ and $F 2$ of 33 male talkers saying "heed" (Peterson \& Barney, 1952); this was also the highest rated stimulus across both the $P$ and NP contexts. The $P$ stimulus was used as the standard (A) for a subsequent $\mathrm{AX}$ discrimination task in a $\mathbf{P}$ context. The discrimination standard for the NP context was a low-rated stimulus (labeled " $N$ " in Figure 1). Discrimination of stimuli in each context was evaluated as a function of distance in perceptual space from the standard. For equal distances in $\mathrm{Mel}$ 


\section{Stimulus Space for C-major Chords}

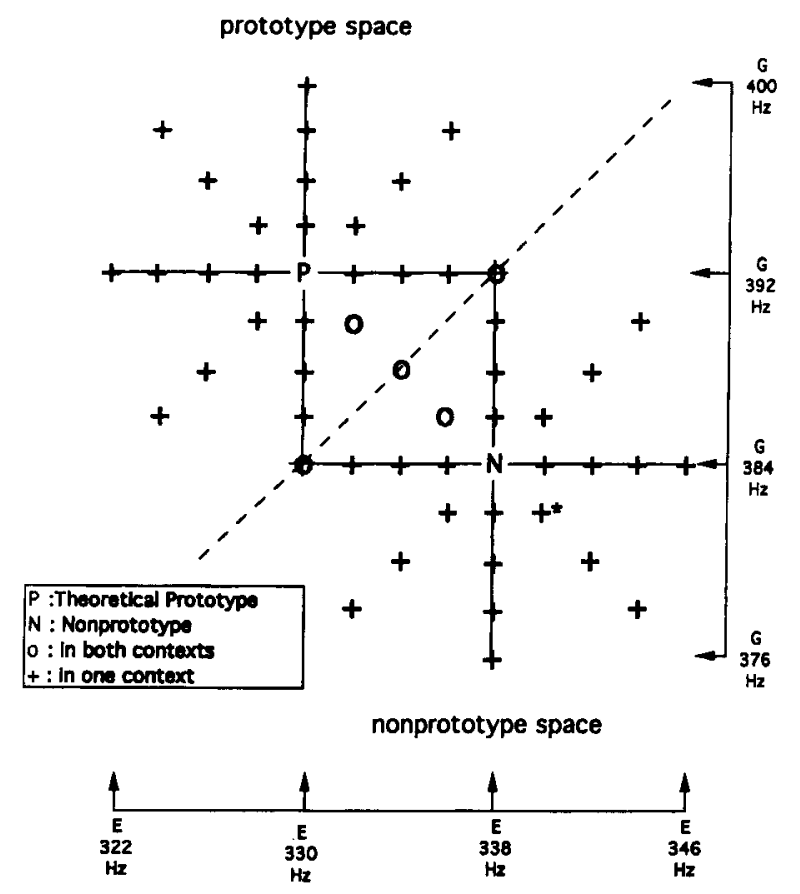

Figure 1. Stimulus space for C-major triads.

units, discrimination was found to be lower for the $\mathrm{P}$ than for the NP standard. This finding was interpreted as reflecting reduced discrimination in the region of the prototype, with the prototype acting as a type of perceptual magnet that reduces the perceived distance of neighboring stimuli.

\section{Prototype and Exemplar Notions}

Kuhl's original research has been criticized for its apparent focus on prototypes, rather than exemplars (P. Kuhl, personal communication to the second author, May 1992). Prototype and exemplar models are the two predominant approaches to modern conceptualizations of perceptual categorization, each providing a different assumption about the nature of category membership. Prototype models (see, e.g., Posner \& Keele, 1968) attribute categorization to the comparison of incoming stimuli with internal prototypes, which are some form of averaged or ideal category representations. Although the exact nature of a prototype may differ across models, there is some agreement that experience with a particular category contributes to defining (or activating) the category prototype, and that categorization is determined by the relative match between the incoming stimulus and the prototype. As similarity to the prototype decreases, the quality of category membership decreases, and the probability of assignment to the given category should also decrease. Furthermore, as stimuli increase in similarity to the prototypes for other categories, the probability of assignment to other categories should increase. It has often been argued that cate- gorization findings are described better by exemplar, rather than prototype, models. Exemplar models (see, e.g., Medin \& Schaffer, 1978; Nosofsky, 1991) propose that experiences (exemplars) are stored in memory and that categorization is determined by the set of exemplars elicited by the incoming stimulus. Exemplars for a given category thus are specific instances of a stimulus, rather than a single, averaged representation of experienced or idealized stimuli. In the modeling of categorization, the incoming stimulus is assumed to be categorized according to memory-retrieval processes which are a direct function of the degree of similarity to the stored exemplars. If similarity is high in relation to the pool of exemplars for a given category, the incoming stimulus will be assigned to that category. However, most research on exemplars has focused on limited, often artificially defined, categories. Furthermore, Li and Pastore (1992) note that differentiating between exemplar and prototype models for natural categories (e.g., stop consonants) can be very difficult (see also Nosofsky, Palmeri, \& McKinley, 1993).

Although the differences between exemplar and prototype notions are theoretically important for understanding categorization processes, the more global level of analysis in the present manuscript does not attempt to differentiate between the two models. Specifically, our research strategy and our predictions are independent of whether stimuli are analyzed in terms of exemplars (i.e., a set of probability density functions) or prototypes (e.g., central tendencies of past experiences or expectations). As a result, although the term prototype will be used throughout this paper, it is understood that subjects could be evaluating stimuli by sampling from a pool of stored exemplars rather than by comparison with a prototype.

Predictions, ratings. Should the consistent goodness ratings for the shared stimuli, as reported by Kuhl (1991), be expected? The goodness rating for a specific stimulus is usually based on comparisons with the entire range of stimuli used in the given rating condition (Parducci \& Perret, 1971). The qualitative range and distribution of stimuli should not be equivalent for the $P$ and NP sets of stimuli. On the basis of the distribution of stimuli in Figure 1, the set centered on "P" could be conjectured to have a symmetric distribution of goodness, with a range only between the P and NP stimuli. The set centered on "N" should have a skewed distribution of goodness, with a range from the theoretical prototype ("P") to extremely poor stimuli in the lower right corner of the space. Thus, the NP stimuli have a broader range of goodness than do stimuli in the $\mathbf{P}$ context, with a higher incidence of poor category members. Furthermore, stimuli in a $\mathrm{P}$ context should reflect considerable prior learning, because they include common, frequently experienced representatives of the category. In contrast, since most stimuli from an NP context would not reflect any significant degree of prior knowledge and learning, they are poor representatives of a category and are not often encountered. Therefore, although the two sets of stimuli in the present study are equal in the size of physical range, the distribution of perceived goodness should not be equal, and these differ- 
ences in the qualitative distribution of the stimuli should be reflected in the ratings of goodness (Parducci \& Perret, 1971).

In following the basic pattern of stimuli used in the Kuhl (1991) study, a small subset of stimuli (the P and N stimuli plus the circles in Figure 1) in the present study were common to both the $\mathrm{P}$ and NP stimulus sets. The differences in qualitative distributions across the contexts should result in the differential use of rating scales, which should be reflected in the ratings assigned to the stimuli common to the two contexts. In particular, a stimulus rated relatively low in a $\mathrm{P}$ context should be rated higher if presented in an NP context, where it is relatively better than the other members of the NP stimulus set.

Discrimination. In contrast to the differences expected in the goodness ratings between the P and NP contexts, there are no compelling a priori reasons for expecting discrimination to be either enhanced or depressed near the prototype. This point can be illustrated by deriving opposite predictions from two simple models, which differ in terms of assumptions about the basic information used in discrimination. For the two models, it is assumed that discrimination is described by a Weber's law relationship ( $\Delta I=\mathrm{k}^{*} \mathrm{I}$, where $k$ is a constant). The models are illustrated in Figure 2. The top portion of the figure represents a sample of stimuli varying in physical location relative to a functional prototype. The $P$ stimulus is a discrimination standard that is in close proximity to a functional prototype. Discrimination is to be measured between $\mathrm{P}$ and $\mathrm{S} 1$, which are separated by a physical distance, $\Delta d_{\mathrm{c}}$. The $\mathrm{N}$ stimulus is a discrimination standard which is far from the prototype, yet within the same perceptual category. Discrimination is to be measured between $\mathrm{N}$ and $\mathrm{S} 2$, which are separated by the same physical distance, $\Delta d_{c}$.

The lower panels of Figure 2 represent a Weber relationship for two different values of $I$ (left panels). The corresponding values of $\Delta l$ (right panels) are based on a
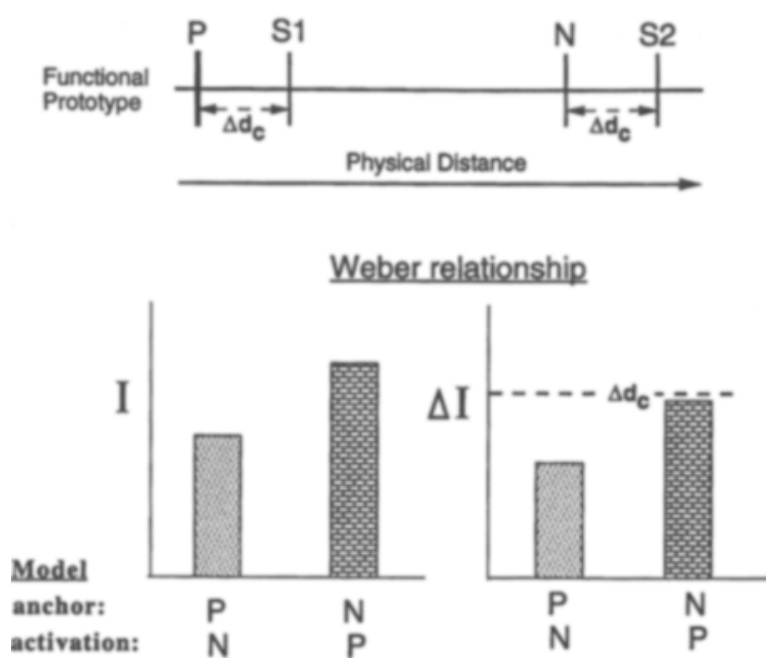

Figure 2. Predictions based on two different applications of Weber's Law (anchor or degree of activation). single Weber constant. The two models are differentiated in terms of the labels for the abscissa of these lower, Weber-relationship panels. The first model (upper abscissa labels prefaced by "anchor") assumes that discrimination is a function of physical dissimilarity between the discrimination standard ( $\mathrm{P}$ or $\mathrm{N}$ ) and a functional prototype which functions as an anchor (e.g., Helson, 1948). ${ }^{2}$ In this conceptualization, perceptual distance, $I$, is assumed to be a monotonic function of physical differences from the prototype. Thus, the values of $I$ and $\Delta I$ are larger for the $\mathrm{N}$ standard in the lower panels of Figure 2. The physical stimulus difference to be discriminated, $\Delta d_{\mathrm{c}}$, is also plotted in the lower right panel. Discrimination performance is a function of the degree to which $\Delta d_{\mathrm{c}}$ exceeds threshold, $\Delta I$. The value of $\Delta d_{\mathrm{c}}$ exceeds the $\mathrm{P}$ threshold by a greater amount than it does the $\mathrm{N}$ threshold. Thus, discrimination based on a prototype functioning as an anchor predicts better discrimination near the prototype, with perceptual distance appearing to be increased around the prototype.

In the second model (lower abscissa labels prefaced by "activation"), discrimination is assumed to be based primarily on degree of activation of a higher level node in a connectionist network. Activation of this higher level node is based on the magnitude of input from lower level nodes which represent features of the stimulus (e.g., F1 and F2 for vowels). Activation of the featural nodes is based on similarity of the component dimensions. In this conceptualization, the degree of activation, $I$, should be greater for stimuli more similar to the prototype. Thus, for the activation model, both $I$ and $\Delta I$ are greater for the $P$ than for the $\mathrm{N}$ standard, and therefore opposite to those for the anchor model. With $\Delta d_{\mathrm{c}}$ closer to the threshold of $\mathrm{P}$ than to that of $\mathrm{N}$, the activation model predicts discrimination to be poorer near the $P$ standard. Therefore, there is no simple, compelling a priori reason to expect enhanced or depressed discrimination in the region of the prototype. ${ }^{3}$

Current research. Several (yet unpublished) attempts have been made to replicate the perceptual magnet effect for vowels. In contrast to Kuhl's (1991) original results, goodness ratings have been found to differ across both subjects and context (Lively, 1993). These results are consistent with the expected difference in qualitative distributions of stimuli in the $\mathrm{P}$ and NP contexts. Lively also found, in contrast to a magnet effect and consistent with the anchor model, slightly heightened discrimination around a single, averaged prototype standard. When individual prototypes were used as the prototype standard instead, discrimination was equal across contexts. LaucknerMorano and Sussman (1993) partially replicated Kuhl's study, but they questioned whether the results reflected a special or a new effect. An identification task was also run, and subjects labeled the $\mathrm{N}$ stimulus as $/ \mathrm{i} /$ only $8 \%$ of the time. Other categories used included /I/, /E/, and /e/. These labeling results suggest that Kuhl's NP stimuli encompassed more than one category, and that the enhanced discrimination in the NP context could possibly reflect the well-known category boundary effect (Pastore, 1976). 
Therefore, the perceptual magnet effect could have been due to artificially enhanced discrimination in the NP context and not to reduced performance in the $P$ context. In more recent work, Davis and Kuhl (1994) and Iverson and Kuhl (1994) have used multidimensional scaling to investigate the perceptual space for the consonants $/ \mathrm{k} / / \mathrm{g} /, / \mathrm{r} /$, and $/ 1 /$. Their results are claimed to show a shrunken perceptual space near the best exemplars of the categories, with the perceptual magnet effect occurring for consonants as well as vowels. These various unpublished studies present conflicting results as to whether or not the perceptual magnet metaphor accurately describes the function of prototypes within categories.

Following the general logic and procedures of the previous speech studies, in the present research we attempted to evaluate qualitative grading and discrimination in a nonspeech category. Prior research has demonstrated that a number of speech phenomena, such as categorical perception (Burns \& Ward, 1978; Locke \& Kellar, 1973; Siegal \& Siegal, 1977) and duplex perception (Hall \& Pastore, 1992; Pastore, Schmuckler, Rosenblum, \& Szczesiul, 1983), also exist for musical stimuli. Although nonspeech stimuli cannot be used to address the validity of speech results directly, nonspeech results can provide an assessment of perceptual processes that may, or may not necessarily, be unique to speech. The results of the present study are therefore important for the evaluation of the general nature of categorization for a natural category and as a comparison for the speech work. In addition, the results address important aspects of music perception, such as the need for sensitivity to fine tuning distinctions.

The present work should be contrasted with earlier categorical perception research in which musical categories were investigated, but in which the focus was on measuring discrimination between rather than within categories. Zatorre and Halpern (1979) evaluated labeling and discrimination for a musical continuum of a simultaneous pair of notes ranging between a major and a minor third. The labeling results for musicians exhibited two categories separated by a relatively sharp boundary. Patterns of discrimination roughly paralleled that predicted from the labeling results, with a peak at the labeling boundary. Burns and Ward (1978) evaluated categorical perception for melodic (sequential) intervals. They found relatively discrete categories with sharp labeling functions. Differences in discrimination for within- and between-category comparisons were evaluated by using several different procedures. With an adaptive procedure (but not with the method of constant stimuli), the contrast between withinand between-category discrimination disappeared with practice.

Although some of these earlier studies did evaluate within-category discrimination, the goal was to provide a contrast with between-category discrimination. These studies thus did not not carefully evaluate changes in discrimination as a function of location within the category. We used goodness ratings and discrimination to investigate the within-category structure of a musical category.

\section{GENERAL METHOD}

\section{Subjects}

An assumption of many types of prototype models is that experience is needed for the formation or activation of good category representations. If knowledge of a particular category is minimal, experience with the category is probably insufficient for a prototype to have been formed. Similarly, exemplar theory requires experience for the development of a reasonable pool of exemplars. Therefore, one must use musically trained subjects. Our 5 subjects, from the SUNY Binghamton community, had a minimum of 10 years of experience with Western music. Two of these subjects had formal, collegelevel training. The subjects were paid $\$ 5 / \mathrm{h}$ for their participation.

\section{Stimuli}

The sampling of stimuli followed that used by Kuhl (1991) for speech stimuli, as described earlier and summarized in Figure 1. Two sets of stimuli were constructed by generating individual sine tones (12-bit, 10-kHz sampling rate) and digitally mixing them to form triads based on a root position C major triad (see Figure 1). In Figure 1, the $30 \mathrm{P}$ stimuli are those above the dashed diagonal line, plus those below the line designated as "o" and "N." The theoretical prototype stimulus ("P" in Figure 1) was an equal-tempered $\mathrm{C}$ major triad $(\mathrm{C}=262 \mathrm{~Hz}, \mathrm{E}=330 \mathrm{~Hz}, \mathrm{G}=392 \mathrm{~Hz})$. The other stimuli were generated by holding the $C$ frequency constant and varying the $E$ and $G$ frequencies in both sharp (increasing) and flat (decreasing) directions in 2- $\mathrm{Hz}$ increments. For 8 of the 30 stimuli, only the $\mathrm{E}$ frequency varied (horizontal axis passing through "P" in Figure 1). For another 8 stimuli, only the $G$ frequency varied (vertical axis passing through "P" in Figure 1). The remaining stimuli fell along the positive and negative diagonals intersecting at " $\mathrm{P}$ " and were created by varying the $E$ and $G$ frequencies simultaneously with equal steps in either the same (both flat or sharp) or opposite (one flat, the other sharp) directions. Stimuli along the negative diagonal included the "N" stimulus, but did not extend beyond it (see Figure 1). The stimuli in the $\mathrm{P}$ context ranged from 322 to $338 \mathrm{~Hz}$ for $\mathrm{E}$ and from 384 to $400 \mathrm{~Hz}$ for $\mathrm{G}$.

In Figure 1, the $30 \mathrm{NP}$ stimuli are those below the dashed diagonal line plus those above the line that are marked "o" and "P." These stimuli were created in the same manner as were the P stimuli, but they were based on a mistuned $\mathrm{C}$ major triad ("N" in Figure 1; $\mathrm{C}=$ $262 \mathrm{~Hz}, \mathrm{E}=338 \mathrm{~Hz}, \mathrm{G}=384 \mathrm{~Hz}$ ) and ranged from 330 to $346 \mathrm{~Hz}$ for E, and 376 to $392 \mathrm{~Hz}$ for G. Summarized in Figure 1, these procedures were used to generate the 30 stimuli differing in equal steps for the prototype and the nonprototype space. Of the 30 stimuli in each set, 7 stimuli (the 5 "o" stimuli plus the "P" and "N" stimuli in Figure 1) occurred in both sets. The complete set of stimuli was constructed to encompass only one perceptual category. Thus, although the NP stimuli were significantly out of tune, the mistuning of both the $\mathrm{E}$ and $\mathrm{G}$ frequencies ensured that the stimuli did not approach another chord category.

Because of the limited frequency range, equal changes in frequency provided a close approximation to equal changes in psychophysical distance. Therefore, the cents scale was not needed to equate perceptual distance. All stimuli were $1,000 \mathrm{msec}$ in duration, were low-pass filtered at $4 \mathrm{kHz}$, and were presented over TDH- 49 earphones at $78 \mathrm{~dB}(\mathrm{~A})$ in commercial sound chambers.

Our musical rating task maintained a within-subjects design, with each subject providing separate ratings for $P$ and NP sets of stimuli (Experiment 1) and then performing separate discrimination tasks (Experiment 2). By using a within-subjects design, we avoided potential problems associated with averaging over legitimate individual differences. For example, if ratings were averaged across subjects to determine a prototype standard, the averaged standard might not be representative of an individual's prototype, and the subsequent discrimination results might not accurately reflect an individual's category structure in relation to this prototype. Empirical evi- 
dence supporting the validity of this concern comes from Lively (1993), where, as noted earlier, the pattern of discrimination differed slightly, depending on whether an averaged prototype or the individual's prototype was used as a standard. In the present study, we thus used individual prototype standards in a discrimination task, and the results should therefore accurately reflect individual category structure.

\section{EXPERIMENT 1}

The goal of Experiment 1 was to use goodness ratings to evaluate qualitative grading in a musical category. In addition, each individual's highest rated stimulus was used to identify his/her prototype standard for the subsequent discrimination task (Experiment 2).

\section{Method}

Subjects were instructed to rate the goodness of each stimulus as a major chord on a scale of 1 (very poor) to 7 (very good). Ratings were indicated by buttonpresses on a telephone keypad and were collected by computer. To become familiar with the stimulus range, subjects listened to the 30 stimuli from a given context ( $P$ or NP) once in random order without responding. To provide practice using the scale, all stimuli were then presented three more times with subjects now responding. Data finally were collected from 20 randomized repetitions of the stimulus set. The procedure was then repeated for the other context, with context order counterbalanced across subjects. The relative magnitude of stimulus ratings for a given context was not a function of order of context evaluation, so order is ignored in the analysis and discussion of results.

\section{Results and Discussion}

As expected, subjects differed considerably in the average and range of ratings both within and between the $P$ and NP contexts (reflecting differences in the use of the rating scale). Thus, the simple pooling of results across subjects would not be indicative of either central tendencies or the range of ratings. In order to better equate ratings across subjects, individual ratings for the $\mathbf{P}$ context were normalized to yield a mean rating of 3.5 (center of rating range) and a standard deviation of 1.8 (selected to keep all ratings between 1 and 7). The individual data for the NP context were normalized for a mean of 2.03 (thus equating the rating of the theoretical prototype stimulus ["P" in Figure 1] across the two contexts) and a standard deviation of 1.09 (the original $S D$ average across all subjects in the NP context). These normalized data were then averaged to yield the rating results shown in Figure 3. While the normalized results do not accurately reflect obtained differences between the rating of the theoretical prototype between the two contexts, they do reflect the relative rating tendencies both within and across contexts, with stimuli in the NP context being rated much lower than those in the $P$ context.

Figure 3 is organized in a fashion which parallels Figure 1, but the shared stimuli are separated. The ratings for stimuli in the $\mathrm{P}$ context are in the left set of bar graphs, with the E frequency along the abscissa and the $G$ frequency along the ordinate. The $\mathrm{P}$ standard is in the center of the set and the $\mathrm{N}$ stimulus is in the lower right corner (with a minimal average rating). Within the data set, the fully shaded stimuli are unique to the $P$ context, with the patterned bars corresponding to the shared stimuli ( $\mathrm{P}, \mathrm{N}$, and o's in Figure 1). The bar for each stimulus represents the average rating within the context, with the standard error bar also plotted. Ratings with nonoverlapping standard error bars are significantly different (Loftus, 1993).

The goodness ratings within the $P$ context declined systematically from the region of the prototype $[F(4,16)=$ $38.38, p<.0001]$. The stimulus with the highest rating for a given subject is assumed to approximate the individual's actual prototype, and this was expected to correspond to the theoretical prototype, the $P$ stimulus. An unexpected finding was that the highest average ratings were never those for the $\mathrm{P}$ stimulus. For all subjects, the average ratings for the stimuli immediately to the left (flat for $\mathrm{E}$ ) and/or above (sharp for $G$ ) the P stimulus were higher than those for the theoretical prototype. With the exception of 2 subjects who exhibited high ratings for one other stimulus $(E+2, G+2$ $\mathrm{Hz}$, and thus sharp for $\mathrm{E}$ as well as $\mathrm{G}$ ), the only stimuli to exceed (or even approach) the ratings of the $P$ stimulus were in this region. The average ratings reflect this distribution of stimuli which are higher in quality than the theoretical prototype; many of these stimuli are significantly higher (more than two standard errors) in rated quality than the $P$ stimulus. Thus the location of the functional prototype is probably somewhat flat for $\mathrm{E}$ and somewhat sharp for $\mathrm{G}$.

There was some variation across subjects in the specific location of the stimulus with the highest rating, with no two subjects exhibiting the identical functional prototype. Table 1 summarizes the frequency characteristics of the individual prototypes which were used as the discrimination standards for the P context in Experiment 2. Table 1 also specifies the unscaled ratings of the individual and theoretical prototype. An analysis of variance (ANOVA) comparing individual and theoretical prototype ratings confirmed that differences were significant $[F(1,4)=22.17, p<.01]$. Defined in terms of the median frequencies from these individual prototypes in Table 1, the most typical functional prototype is slightly flat for $\mathrm{E}(328 \mathrm{~Hz}$ instead of $330 \mathrm{~Hz})$ and slightly sharp for G (394 instead of $392 \mathrm{~Hz}$ ). However, it should be noted that the other stimuli which were flat for E, sharp for $\mathrm{G}$, or both, often received ratings which were not significantly lower than the one meeting the criterion

Table 1

Individual and Theoretical Prototype Ratings in a Prototype Context

\begin{tabular}{|c|c|c|c|c|c|}
\hline & \multicolumn{5}{|c|}{ Subject } \\
\hline & 1 & 2 & 3 & 4 & 5 \\
\hline \multicolumn{6}{|c|}{ Individual Prototype Stimulus } \\
\hline E frequency & $330 \mathrm{~Hz}$ & $328 \mathrm{~Hz}$ & $330 \mathrm{~Hz}$ & $328 \mathrm{~Hz}$ & $328 \mathrm{~Hz}$ \\
\hline G frequency & $396 \mathrm{~Hz}$ & $390 \mathrm{~Hz}$ & $394 \mathrm{~Hz}$ & $392 \mathrm{~Hz}$ & $394 \mathrm{~Hz}$ \\
\hline Rating & 5.3 & 5.9 & 6 & 4.9 & 5.9 \\
\hline \multicolumn{6}{|c|}{ Theoretical Prototype Stimulus } \\
\hline E frequency & $330 \mathrm{~Hz}^{*}$ & & & & \\
\hline$G$ frequency & $392 \mathrm{~Hz}^{*}$ & & & & \\
\hline Rating & 4.3 & 5.6 & 5.1 & 4.1 & 4.5 \\
\hline
\end{tabular}

*Same for all subjects. 

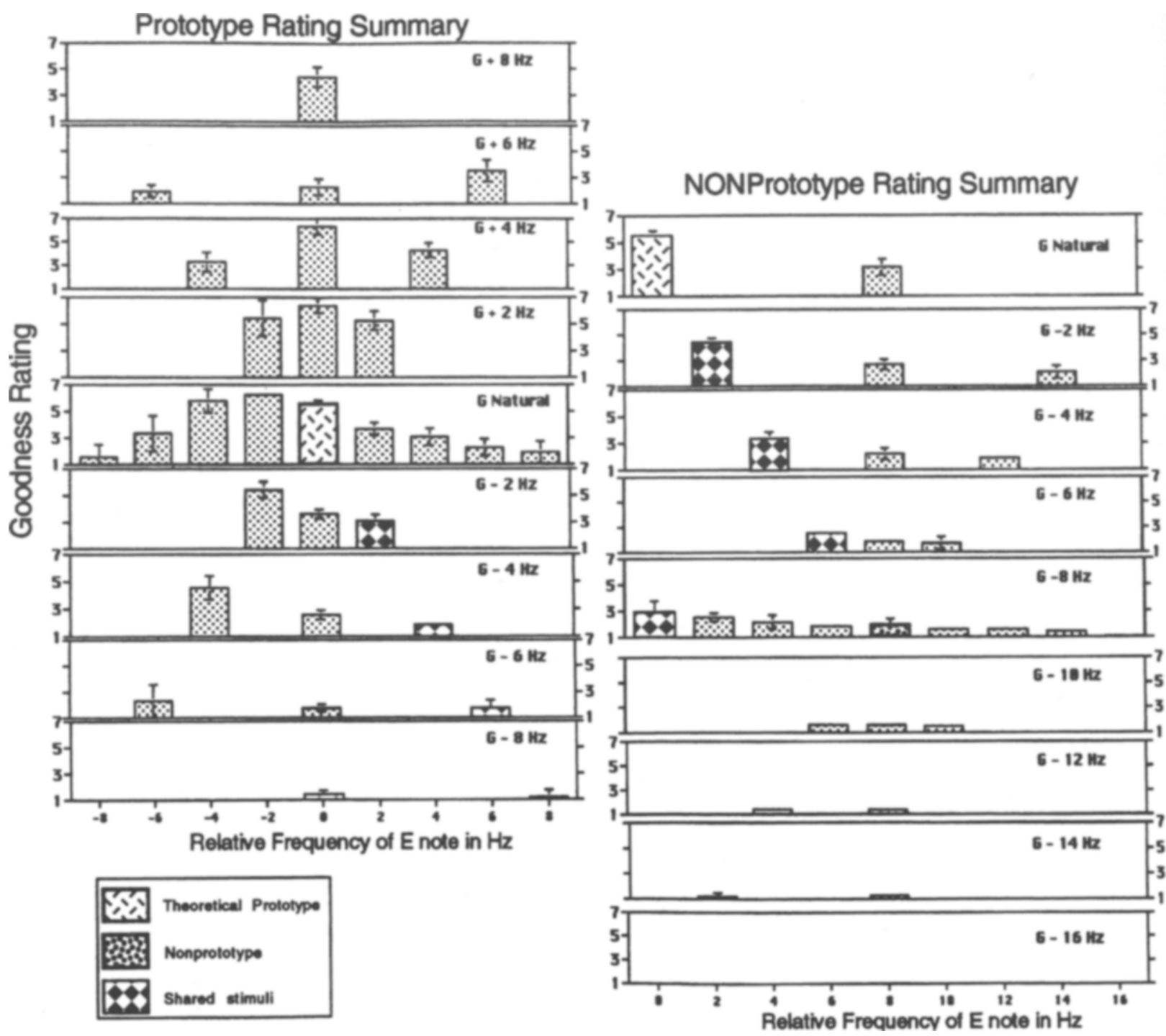

Figure 3. Normalized mean goodness rating summary for the prototype and nonprototype contexts. This figure parallels the structure of Figure 1, with the shared stimuli equivalent to the stimuli represented by "P," “N," and circles in Figure 1.

for the functional prototype. Thus, the frequency properties summarized in Table 1 should only be indicative of the approximate location of the functional prototype. The absence of perfect tuning in each individual's prototype was not completely surprising; several studies have shown that musical intervals are seldom ideal but are often compressed (Rakowski, 1976) or stretched (Hartmann, 1993; Ward, 1954). However, the discrepancy between the functional and theoretical prototypes also could reflect the relative importance of different tuning systems.

Beyond the discrepancy between the functional and theoretical prototypes, the overall distribution of ratings in the $\mathrm{P}$ context (Figure 3 ) may provide information about the differential importance of chord components in defining the major chord prototype. Ratings were relatively high for stimuli maintaining an in-tune $\mathrm{E}$ component with only the $\mathrm{G}$ component varying in a sharp direction. In contrast, ratings of stimuli maintaining a constant $\mathrm{G}$ com- ponent with the E component varying declined more systematically in both directions. These results could be indicative of the importance of the $E$ frequency in determining the goodness of $\mathrm{C}$ major triads.

The right set of bar graphs in Figure 3 represents ratings from the NP context in a manner that roughly approximates the organization of Figure 1. The vertical location accurately reflects the $G$ frequency, but the $E$ frequency is offset horizontally to separate the $\mathrm{P}$ and NP context ratings. For example, the patterned bar in the upper left corner represents the rating of the P stimulus in the NP context; the rating for the identical stimulus in the $\mathrm{P}$ context is in the center of the $P$ context graphs. The ratings for all NP context stimuli were low, but they systematically improved when moving toward the $\mathrm{P}$ context $[F(4,16)=$ $49.72, p<.0001]$. These results are consistent with the notion that quality of category membership is a function of similarity to a category prototype. 
The average original rating of the theoretical prototype ("P" stimulus) in the P context was 4.73 , but it increased to 5.54 in the NP context. This difference was significant $[F(1,4)=35.61, p<.005]$. The set of stimuli in the NP context included the theoretical prototype but none of the actual, individual prototypes. In the NP context, the theoretical prototype received the highest average goodness rating (5.54); this result is consistent with the "P" stimulus' clearly being the "best" stimulus available in the NP stimulus set. In the P context, the P stimulus was not the "best" stimulus available, and it received a lower rating. Also, consistent with the theoretical predictions described earlier, but in contrast to the context independence reported by Kuhl (1991), the ratings of the other shared stimuli clearly differed according to context, with all shared stimuli receiving a higher goodness rating in the NP context. For example, the average original rating of the central nonprototype stimulus ("N" in Figure 1) was 1.56 in the P context, but it increased significantly to 2.33 in the NP context $[F(1,4)=33.08, p<.005]$. Given the present findings and those reported by others (e.g., Lively, 1993), it appears that contextual differences in ratings should be expected.

Finally, the effort to evaluate the existence of a prototype magnet effect for musical chords requires that we find higher goodness for the P than for the NP stimuli. Clearly, stimuli near the prototype were rated higher than stimuli far from the prototype (e.g., those in the NP context). Thus we had quantified category membership as a precursor to Experiment 2, which was carried out to evaluate discrimination as a function of context.

\section{EXPERIMENT 2}

Experiment 1 demonstrated that major chord categories are clearly qualitatively graded, with one stimulus always receiving a distinctively higher rating than other category members, although the specific optimum stimulus may vary (within a limited range) across individual listeners. Furthermore, the goodness ratings of stimuli decreased in a relatively systematic fashion as a function of distance from the prototype. It can be inferred from these findings that musical categories may be structured around prototypes or frequent exemplars (i.e., the highest rated stimulus). Therefore, a discrimination task similar to that used in recent speech studies (e.g., Kuhl, 1991) should be able to effectively evaluate the function of a prototype in this natural, nonspeech category. We approached Experiment 2 without a specific expectation concerning the pattern of results; the speech discrimination results from several studies were not consistent, and, as summarized above, simple models using a Weber relationship, but based on a perceptual anchor versus activation of a node, make conflicting predictions concerning whether discrimination should be lower or higher in a P context than in an NP context.

\section{Method}

Because the musical rating task revealed individual differences, we used a within-subjects design for the discrimination task, with each subject's highest rated stimulus from the goodness rating task employed as that subject's prototype standard. For most of our sub- jects, the prototype (summarized in Table 1) was within approximately $2-4 \mathrm{~Hz}$ from the perfectly tuned triad (which was the center of the P stimulus set); therefore the NP standard was selected to be slightly offset from the center of the NP stimulus set. Compared with the "N" stimulus in Figure 1, the NP standard contained an E frequency that was $2 \mathrm{~Hz}$ sharp and a $\mathrm{G}$ frequency that was $2 \mathrm{~Hz}$ flat (this discrimination standard is marked with an asterisk in Figure 1). Unlike the P standard, the NP standard had no special significance other than that it represented a control condition, so there was no need to modify the selected standard for individual subjects. Therefore, a single NP standard was used for all subjects.

Because Kuhl (1991) wanted to use a single procedure for human infants and adults, as well as monkeys, she used a go/no-go procedure. A go/no-go task is essentially equivalent to an $\mathrm{AX}$ procedure with only one overt response category. Even with two response categories, the AX procedure is highly sensitive to response bias (Green \& Swets, 1966). Furthermore, a recent study of AX discrimination of stimuli drawn from a continuum between a major triad and its related minor triad (Howard, Rosen, \& Broad, 1992) demonstrated significant bias. Therefore, we used a two-interval forced choice task, which is empirically (see, e.g., Macmillan \& Creelman, 1991), if not also theoretically (see, e.g., Green \& Swets, 1966), bias free and thus should provide a reliable discrimination measure for each stimulus. In addition, we used 28 repetitions for each stimulus as opposed to the 2 used in the Kuhl (1991) discrimination task. With the greater number of trials per subject, performance for each individual stimulus comparison can be accurately evaluated for every subject. With only two trials per subject, data must be pooled across subjects.

The experiment was run in two sessions. Within each session, discrimination for each subject was evaluated in separate $P$ and NP blocks of trials. Selection of initial context ( $P$ or NP) was counterbalanced across subjects, and the context initially presented to the subject in the first session was presented last in the second session. On each trial, subjects heard two pairs of triads, consisting of a same and a different pair. In each pair, the standard always was presented first. In same pairs, the given standard was repeated. The second stimulus in the different pair was randomly selected from the 29 other triads. For example, the sequence of stimuli on a trial with the difference in the second pair would be: standard, standard; standard, comparison. The ordering of pairs within a trial was also randomized. The subjects indicated by a buttonpress which pair contained the different triads.

\section{Results and Discussion}

Discrimination results for both contexts are shown in Figure 4. Because individual prototypes (not the centrally located "P" stimulus in Figure 1) were used in the P context, it is not possible to present the data in a systematic fashion like that in Figure 3 or like that employed by Kuhl (1991), with discrimination relative to a fixed standard represented in terms of absolute stimulus location (e.g., see our Figure 1). With the prototype standard varying between our subjects, we must represent the data in terms of distance from the individuals' prototype; in order to keep the discrimination summary relatively simple, we used city block distances. ${ }^{4}$ Therefore, the data from the $\mathrm{P}$ context contributing to each of the bars for the largest distances do not necessarily represent all subjects. ${ }^{5}$ For the NP context, the same standard was used for all subjects, and thus each bar contains data from all subjects. The standard error bars around each mean can be used to evaluate the statistical significance of the differences.

In contrast to the results reported for speech categories (see above), discrimination for our musical triads was higher in the $\mathrm{P}$ context than in the NP context. In the $\mathrm{P}$ 


\section{Discrimination as a function of distance from standard}

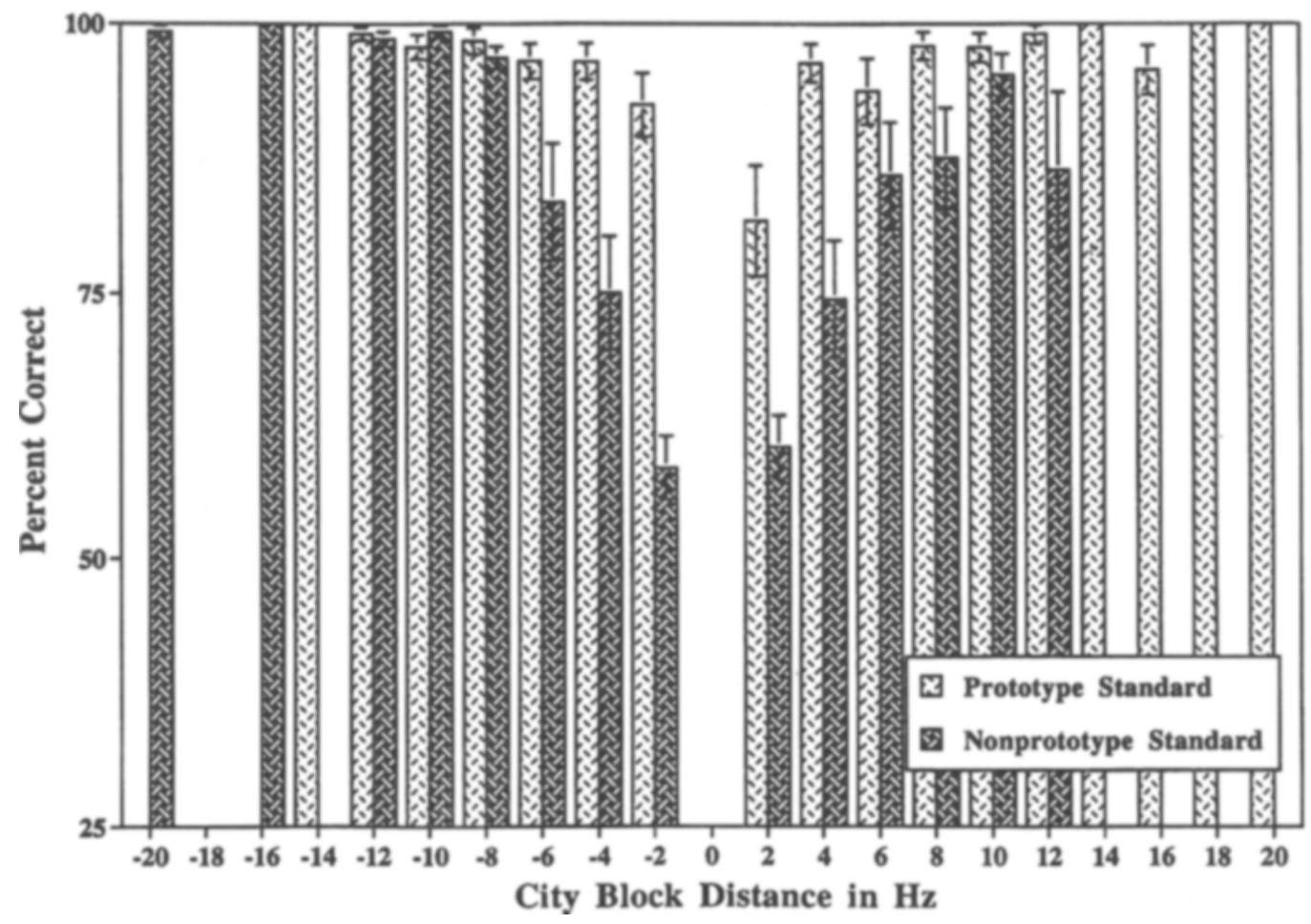

Figure 4. Discrimination as a function of city block distance from a standard (see text).

context, average discrimination at $2 \mathrm{~Hz}$ from the functional prototype of the major triad was $82 \%$ when stimuli changed in a positive (musically sharp) direction and at 93\% when stimuli changed in a negative (musically flat) direction. In the NP context, respective discrimination was significantly lower at $60 \%$ and $59 \%$. Although substantial individual differences existed in absolute levels of performance, all subjects showed a similar trend, with average performance jumping to near perfect performance for a difference of $4 \mathrm{~Hz}$ from the P standard. In fact, 1 subject demonstrated virtually perfect discrimination in the $\mathrm{P}$, but not the NP, context. Differences in discrimination between the contexts were significant for smaller distances, but (as expected) were not significant at larger distances where performance was at ceiling. All of these findings are consistent with the predictions from a Weber relationship model based on a perceptual anchor rather than degree of activation.

\section{GENERAL DISCUSSION}

In the present study, we investigated aspects of perception for musical triads varying systematically in the frequency of two steady-state components. Goodness ratings revealed that the theoretical musical prototype (the equaltempered triad) was never rated the highest in the $\mathrm{P}$ context. Stimuli that were flat for the $E$ frequency, sharp for the $\mathrm{G}$ frequency, or both were consistently rated higher than the theoretical prototype, with the highest rated stim- ulus, or functional prototype, falling within this stimulus range. Furthermore, in contrast to the previous results for vowels, all stimuli common to both the $P$ and NP sets $(P$, $\mathrm{N}$, and o's in Figure 1) were rated higher in the NP context. For both individual and pooled results, discrimination for equal distances from a standard in a musical category was better in a P context than in an NP context. Although in contrast to a perceptual magnet effect, these discrimination findings from a natural category are consistent with predictions from a model in which a prototype is functioning as an anchor. These results have a number of implications for our understanding of music perception as well as provide an empirical nonspeech contrast to existing speech findings.

\section{Implications for Music Perception}

Several musical factors may have contributed to the present pattern of findings. Superior discrimination in a $P$ context is very functional for musicians. In an ensemble situation, sensitivity to fine tuning distinctions is imperative, because only small deviations from in-tune intervals can be tolerated before dissonance is perceived within the aggregate of ensemble members. Dissonance (or lack of dissonance) certainly may provide a basis for one level of discrimination, as well as limit the range of high goodness for a category. Extensive experience with a sampling of stimuli from a small range of deviations around in-tune intervals (the essence of categorization based on exemplars) should also provide a basis for the formation (or activa- 
tion) of a strong prototype. In contrast, musicians generally neither hear nor play extremely out-of-tune (dissonant) stimuli. This lack of experience with the majority of NP stimuli would probably not allow the formation (or activation) of a prototype. If discrimination is achieved with some form of a prototype, these experiential differences should result in overall enhanced discrimination in a $P$ context, but not in an NP context.

In the present study, discrimination in the $P$ context was relative to the operationally defined (highest rated) functional prototypes. The stimulus representing the functional prototype varied somewhat across individuals and its goodness rating often did not differ significantly from that for other stimuli which were similarly offset from the equal-tempered $\mathbf{P}$ stimulus. Therefore, although our data are based on the mean value from 20 repetitions of each stimulus, some variation within individuals might be expected if the rating task was repeated. Thus, while a small portion of the individual differences could be due to measurement error, it seems clear that all subjects exhibited a somewhat flexible prototype within a range of stimuli other than the one defined by equal temperament.

Several aspects of musical performance appear to require some flexibility in ideal tunings. For example, agreement on the exact width of prototypic intervals can be somewhat determined within an ensemble, with tuning achieved relative to these intervals and not to some ideal internal standard. Tuning in terms of some defined standard becomes even more important with the introduction of an instrument, such as a piano or organ, which has no immediate control over its tuning. In such a situation, prototypic intervals for the ensemble must be made relative to the current tuning of the fixed-frequency instrument and not to some ideal internal standard. Therefore, some flexibility in what constitutes a prototypic interval is necessary if one is to adapt adequately to various musical situations (such flexibility in musicians is not equivalent to an insensitivity to small differences). Such experiences may be one factor contributing to the observed individual differences in prototypes found in Experiment 1.

The prototype context was centered on an equal-tempered major chord, but the highest median goodness ratings were for stimuli that contained a flat $\mathrm{E}$ frequency $(328 \mathrm{~Hz}$ instead of $330 \mathrm{~Hz}$ ) and a sharp G frequency (394 instead of $392 \mathrm{~Hz}$ ). With C specified at $262 \mathrm{~Hz}$, the just-tuned prototype for the $\mathrm{E}$ and $\mathrm{G}$ frequencies should be 327.5 and $393 \mathrm{~Hz}$. Therefore, within the accuracy of the $2-\mathrm{Hz}$ steps between the stimuli in the present study, the ratings appear to reflect a consistent preference for chords tuned according to just temperament.

For practical reasons, most Western tonal music is based on equal temperament, which does not maintain pure ratios between intervals, but allows a musician to play in any key without retuning the instrument. This prevalence of equal temperament in Western music should lead to the type of cultural conditioning of a strong prototype often conjectured in the music literature (e.g., Lundin, 1967; Roederer, 1973). The dominance of this tuning sys- tem is also reflected by the use of this tuning system in most music perception research (e.g., Krumhansl, 1990).

In contrast to equal temperament, just temperament maintains pure interval ratios, which reflects some basic perceptual and physiological aspects of pitch perception (Boomsliter \& Creel, 1961; Moore, 1989). However, because just temperament has a major practical shortcoming (modulation to other keys is not possible), it is not used widely today. Moore (1989) notes that although the ratio differences between the two tuning systems are small, noticeable beats are probably present in equal-tempered intervals (e.g., a perfect fifth has a frequency ratio of $3: 2$, but the ratio is $2.9966: 2$ for an equal-tempered fifth). The apparent preference for the more pure intervals of the justtuned chords demonstrates that even extensive experience with equal temperament may not override prototypes based on physiological aspects of the auditory system. More recent work from our laboratory done with a discrimination task has also shown that individual prototypes are more consistent with just, rather than equal, temperament (Acker, 1994).

Several other aspects of the present findings warrant further investigation. In Experiment 1, relatively high levels of goodness were maintained for a range of frequencies for a constant $E$ component with the $G$ component varying. However, ratings declined more evenly when the $\mathrm{G}$ component was constant and the $\mathrm{E}$ varied. This asymmetry between the $\mathrm{E}$ and $\mathrm{G}$ components of the triad is present even after compensation for the location of the justtuned prototype. Thus, in a $\mathrm{C}$ major triad, there seems to be an asymmetry in the independence of these two components. Effective evaluation of the separability of the frequencies requires the use of discrimination tasks with systematic, symmetric variation in the dimensions (see, e.g., Garner, 1976). Unfortunately, direct or parallel comparisons of the present goodness and discrimination results cannot be made because individual prototypes, rather than the perfectly tuned theoretical prototype, were used as the standard for discrimination in the $P$ context with the fixed set of stimuli centered on the equal-tempered prototype. As a consequence, variation in either $E$ or $G$ also involved changes in the other note. Although such discrimination should be evaluated empirically, we might assume that some differences in discrimination would follow the reported differences in goodness. Such findings would indicate that the $\mathrm{E}$ frequency is probably the more important component in $\mathrm{C}$ major triads.

Research has shown that interval tunings differ as a function of musical context (Shackford, 1961, 1962a, $1962 \mathrm{~b})$. Although the major triads in the present study were rated in isolation, it is possible that ratings would differ slightly if stimuli were presented within a tonal framework, shifting to the tuning system employed in the composition, which, in much of Western music, is equal temperament. In addition, ratings would probably vary somewhat according to the tonal framework in which they were placed (e.g., predictable framework of the baroque period vs. a nonpredictable framework of a 20 th-century 
12-tone row piece). Furthermore, although only a major triad was used in the present study, it also seems logical that the present findings should generalize to other isolated chord types, such as minor, diminished, or seventh chords. However, overall poorer performance might result with less common constructions (e.g., seventh chords), since reduced experience with such chords should result in less representations than would more common constructions, such as the major triad used in the present study. Future work should evaluate these various questions.

\section{Comparison With Recent Speech Studies}

The original motivation behind the present study was the work in which Kuhl (1991) demonstrated a perceptual magnet effect for a vowel prototype. The present results do provide a context in which to evaluate those speech findings. The first obvious difference in results is the individual variation in the location of the functional prototype. The original work of Peterson and Barney (1952) identified the average formant frequencies of vowels on the basis of an analysis of production. Kuhl's results, pooled across adult listeners (but highly consistent across individual listeners), seemed to indicate that the perceptual prototype corresponded to this production mean. However, consistent with the present results for musical triads, later goodness ratings of the vowel stimuli (Lively, 1993) indicate differences in the precise location of individual prototypes. Like the triads, the individual vowel prototypes are confined to a restricted range near the theoretical prototype. More recently, Iverson and Kuhl (1995), using a more limited set of stimuli, reported a skewed distribution of high ratings relative to the $P$ stimulus, with the highest rated vowel stimuli not precisely corresponding (by a small difference) to Peterson and Barney's average. Thus, some individual variability in prototype specification is consistent across speech and music.

For several theoretical reasons (discussed earlier), we had expected context-specific differences in the ratings of stimuli common to both contexts. The present results confirmed these expectations. The absence of such contextrelated differences in Kuhl's (1991) study might have been indicative of differences in processing of speech and music, with the vowel stimuli processed in a more absolute, context-independent fashion. However, Lively (1993) also found that goodness ratings differed across context, with all vowel stimuli common to both $P$ and NP contexts rated higher in the NP context than in the P context. These alternative vowel results are consistent with the theoretical considerations described earlier. However, the magnitude of difference in context-dependent goodness ratings between the present and the Kuhl study may be a function of stimulus selection. In the original Kuhl study, the functional prototype stimulus was present in both the P and the NP sets of stimuli and thus should have provided an anchor at the highest goodness rating; in the present study, the functional prototype was never present in the NP stimulus set. This difference in stimulus distribution might have contributed to differences in the mag- nitude of context-specific ratings of the shared stimuli. However, if the more recent goodness rating results from Iverson and Kuhl (1995) apply to Kuhl's (1991) stimuli, the original NP stimulus set also may not have contained the functional vowel prototype. If each individual's prototype had been present in the NP context, it is possible that this stimulus would have functioned as a perceptual anchor, thus reducing range effects.

The most obvious difference in the results between the present study of musical triads and the Kuhl study of vowels is the pattern of discrimination results supporting anchor versus magnet conceptualizations of prototype function. Kuhl (1991) found better discrimination in an NP context than in a P context and interpreted these results as demonstrating reduced performance in the $\mathrm{P}$ context as opposed to enhanced performance in an NP context. Although such a conclusion is consistent with a Weber relationship based on degree of activation, rather than a perceptual anchor, the validity of this interpretation is based on the logical assumption that, other factors being equal, performance differences should be attributed to the action of the prototype, rather than to some unknown factor enhancing performance in the NP context. This logic leads to the conjectured "perceptual magnet" metaphor. If this assumption is valid, it would seem that music and speech categorization processes are qualitatively different. However, there is some concern that this important assumption may not be valid, with the perceptual magnet effect possibly reflecting artificially enhanced discrimination in the NP context, rather than reduced discrimination in the $P$ context. As noted above, Lauckner-Morano and Sussman (1993) used a labeling task to demonstrate that Kuhl's original NP context may have contained more than one category. An identification task, used in more recent work by Iverson and Kuhl (1995), has also shown the NP stimuli to include the category /e/. If this is an accurate assessment of the original NP context for vowel stimuli, then Kuhl's original findings may not be indicative of a magnet effect, but rather of artificially enhanced discrimination in the NP context due to between-category comparisons. ${ }^{6}$ Thus, additional speech research is necessary, in which the nature of prototype and nonprototype discrimination can be studied under acceptably equivalent conditions. The present results from another natural category, while not directly addressing the validity of the speech findings, effectively map category structure for musical triads and can also serve as a comparison for future speech findings.

\section{REFERENCES}

ACKER, B. E. (1994). Discrimination of musical chord components. Unpublished master's thesis, State University of New York, Binghamton.

Boomsliter, P., \& Creel, W. (1961). The long pattern hypothesis in harmony and hearing. Journal of Music Theory, 5, 2-31.

BURnS, E. M., \& WARD, W. I. (1978). Categorical perceptionphenomenon or epiphenomenon: Evidence from experiments in the perception of melodic musical intervals. Journal of the Acoustical Society of America, 63, 456-468. 
Collins, S. C. (1985). Duplex perception with musical stimuli: A further investigation. Perception \& Psychophysics, 38, 172-177.

Davis, K., \& KuHL, P. (1994, June). Tests of the perceptual magnet effect for American English $/ \mathrm{k} /$ and $/ \mathrm{g} /$. Paper presented at the meeting of the Acoustical Society of America, Cambridge, MA.

GARNER, W. R. (1976). Interaction of stimulus dimensions in concept and choice processes. Cognitive Psychology, 8, 98-123.

GREen, D. M., \& SwETs, J. A. (1966). Signal detection theory and psychophysics. New York: Wiley

HaLl, M. D., \& PASTORE, R. E. (1992). Musical duplex perception: Perception of figurally good chords with subliminal distinguishing tones. Journal of Experimental Psychology: Human Perception \& Performance, 18, 752-762.

HARTMANN, W. M. (1993). On the origin of the enlarged melodic octave. Journal of the Acoustical Society of America, 93, 3400-3409.

HeLson, H. (1948). Adaptation level as a basis for a quantitative theory of frames of reference. Psychological Review, 55, 297-313.

Howard, D., Rosen, S., \& BROAD, V. (1992). Major/minor triad identification and discrimination by musically trained and untrained listeners. Music Perception, 2, 205-220.

IVERSON, P., \& KUHL, P. (1994, June). Tests of the perceptual magnet effect for American English $/ r /$ and $/ / /$. Paper presented at the meeting of the Acoustical Society of America, Cambridge, MA.

Iverson, P., \& KuHL, P. (1995). Mapping the perceptual magnet effect for speech using signal detection theory and multidimensional scaling. Journal of the Acoustical Society of America, 97, 553-562.

KRUMHANSL, C. L. (1990). Cognitive foundations of musical pitch. New York: Oxford University Press.

KUHL, P. K. (1991). Human adults and human infants show a "perceptual magnet effect" for the prototype of speech categories, monkeys do not. Perception \& Psychophysics, 50, 93-107.

Lauckner-Morano, V., \& Sussman, J. E. (1993, May). Identification and change/no-change discrimination of /i/ stimuli: Further tests of the "magnet" effect. Paper presented at the meeting of the Acoustical Society of America, Ottawa, ON, Canada.

Li, X., \& PAStore, R. E. (1992). Evaluation of prototypes in perceptual space for a place contrast. In M. E. H. Schouten (Ed.), The auditory processing of speech (pp. 303-308). New York: Mouton De Gruyter.

LIVELY, S. E. (1993, May). An examination of the perceptual magnet effect. Paper presented at the meeting of the Acoustical Society of America, Ottawa, ON, Canada.

LoCKe, S., \& KelLeR, L. (1973). Categorical perception in a nonlinguistic mode. Cortex, 9, 355-369.

Loftus, G. R. (1993). Editorial comment. Memory \& Cognition, 21, 1-3.

Lundin, R. W. (1967). An objective psychology of music (2nd ed.). New York: Ronald Press.

MaCmillan, N. A., Braida, L. D., \& GoldberG, R. F. (1987). Central and peripheral processes in the perception of speech and nonspeech sounds. In M. E. H. Schouten (Ed.), Psychophysics of speech perception (pp. 28-45). Dordrecht: Martinus Nijhof.

Macmillan, N. A., \& Creelman, C. D. (1991), Detection theory: A user's guide. Cambridge: Cambridge University Press.

Medin, D. L., \& Schaffer, M. M. (1978). Context theory of classification learning. Psychological Review, 97, 225-252.

MOORE, B. J. (1989). An introduction to the psychology of hearing. London: Academic Press.

NosofSKY, R. M. (1991). Tests of an exemplar model for relating perceptual classification and recognition memory. Journal of Experimental Psychology: Human Perception \& Performance, 17, 3-27.

Parducci, A., \& Perret, L. F. (1971). Category rating scales: Effects of relative spacing and frequency. Journal of Experimental Psychology, 89, 427-453.

PAstoRE, R. E. (1976). Categorical perception: A critical re-evaluation. In S. K. Hirsh, D. H. Eldredge, I. J. Hirsh, \& S. R. Silverman (Eds.), Hearing and Davis: Essays honoring Hallowell Davis (pp. 253-264). St. Louis: Washington University Press.

PASTORE, R. E. (1987). Categorical perception: Some psychophysical models. In S. Harnad (Ed.), Categorical perception: The groundwork of cognition (pp. 29-52). New York: Cambridge University Press.

Pastore, R. E., Schmuckler, M. A., Rosenblum, L., \& Szczesiul, R.
(1983). Duplex perception with musical stimuli. Perception \& Psychophysics, 33, 469-474.

Peterson, G. E., \& Barney, H. L. (1952). Control methods used in a study of the vowels. Journal of the Acoustical Society of America, 24, 175-184.

PoSNER, M. I., \& KEELE, S. W. (1968). On the genesis of abstract ideas. Journal of Experimental Psychology, 77, 353-363.

RAKOWSKI, A. (1976). Tuning of isolated musical intervals. Journal of the Acoustical Society of America, 59, S50.

ROEDERER, J. G. (1973). Introduction to the physics and psychophysics of music. London: English Universities Press.

Samuel, A. G. (1982). Phonetic prototypes. Perception \& Psychophysics, 31, 307-314

SHACKFORD, C. (1961). Some aspects of perception. I. Journal of Music Theory, 5, 162-202.

SHACKFORD, C. (1962a). Some aspects of perception: II. Journal of Music Theory, 6, 66-90.

SHACKFORD, C. (1962b). Some aspects of perception: III. Journal of Music Theory, 6, 2953

Siegal, J. A., \& Siegal, W. (1977). Categorical perception of tonal intervals: Musicians can't tell sharp from flat. Perception \& Psychophysics, 21, 399-407.

StudDerT-Kennedy, M., Liberman, A. M., Harris, K. S., \& COOPER, F. S. (1970). Motor theory of speech perception: A reply to Lane's critical review. Psychological Review, 77, 234-249.

Volatis, L. E., \& MrlleR, J. L. (1992). Phonetic prototypes: Influence of place of articulation and speaking rate on the internal structure of voicing categories. Journal of the Acoustical Society of America, 92, 723-735.

WARD, W. D. (1954). Subjective musical pitch. Journal of the Acoustical Society of America, 26, 369-380.

ZATORRE, R. J., \& HALPERN, A. R. (1979). Identification, discrimination, and selective adaptation of simultaneous musical intervals. Perception \& Psychophysics, 26, 384-395.

\section{NOTES}

1. We are defining a category as natural in the sense that it occurs frequently and can be culturally defined. By utilizing major chords, in the present experiments we investigated Western musical categories.

2. Helson (1948) predicts an experience-based standard located at a category boundary as opposed to within the category. More recent conceptualizations of prototypes often have used similar notions of prototypes which function instead as central anchors.

3 . Both the anchor and activation models assume that a constant physical change results in a constant perceptual change, and thus the stimulus difference to be discriminated, $\Delta d_{\mathrm{c}}$, is independent of $I$. An alternative assumption for both models is that Weber's law also applies to the physical difference between the stimuli to be discriminated and thus is proportional to $I$ and, as a result, also $\Delta I$ (i.e., $\Delta d=k_{1}{ }^{*} I ; \Delta I=k_{2}{ }^{*} I$ $\Delta d=k_{3}{ }^{*} \Delta I$ ). Applying this alternative assumption to either model results in the prediction of equal performance for both the $\mathrm{P}$ and the NP standards. Thus, the simple discrimination models outlined in Figure 2 , as well as variations on such models, allow prediction of all possible outcomes.

4. City block distances sum the separate values along the major dimensions, whereas Euclidean distances reflect the shortest straight-line distance. In Figure 1, the $\mathrm{P}$ and $\mathrm{N}$ stimuli differ by $8 \mathrm{~Hz}$ along both the $\mathrm{E}$ and $\mathrm{G}$ dimensions. The respective city block and Euclidean distances between the stimuli are 16 and $11.3 \mathrm{~Hz}$.

5 . With a different location of prototype standards for individual subjects, and a fixed set of stimuli for the $\mathbf{P}$ context, there are differences in the extreme city block distances for discrimination. In Figure 1, if the individual prototype is located immediately to the left of $P(-2 \mathrm{~Hz}$ in $\mathrm{E}$, same $\mathrm{G}$ ), the most distant stimulus, $\mathrm{N}$, differs by $18 \mathrm{~Hz}$. If the individual prototype is also $2 \mathrm{~Hz}$ sharp in $\mathrm{G}(-2 \mathrm{~Hz}$ for $\mathrm{E},+2 \mathrm{~Hz}$ for $\mathrm{G})$, the city block distance to $\mathrm{N}$ is $20 \mathrm{~Hz}$.

6. There have been conjectures that the perceptual magnet effect for vowels may simply reflect the categorical perception of speech stimuli (see, e.g., Iverson \& Kuhl, 1994; Lauckner-Morano \& Sussman, 1993). Although in the classic categorical perception literature it had been 
found that vowels were considerably less categorical, and more variable in identification, than consonants (e.g., Studdert-Kennedy et al., 1970), vowels still should exhibit a category boundary effect (enhanced discrimination at and near the category boundary relative to within categories). Since categorical perception results for chords should be roughly equivalent to that reported for vowels (Collins, 1985; Pastore et al., 1983), why did we not find a similar perceptual magnet effect in the present study? This one, apparently simple, question raises many issues.

In categorical perception, why is discrimination enhanced at and near the category boundary relative to within categories? One conjecture is that there is a threshold (see, e.g., Pastore, 1987) or perceptual anchor (Macmillan, Braida, \& Goldberg, 1987) at the category boundary, thus accounting for category boundary effects. Although this conjecture may be valid in some circumstances, it is likely that categorization is often based on a prototype which, by definition, should be internal to the category, and not at the category boundary. How can such a prototype function as an anchor and yet be compatible with category boundary effects? The classification of speech (and possibly other types of stimuli, such as music) may be relatively automatic and based on the use of prototype (or exemplar) information, with the higher level categorization then serving as a basis for enhanced discrimination for comparisons across categories. Macmillan et al. (1987) provide an analysis of vowel discrimination results which support the latter conjecture, concluding that the category boundary effect for vowels is "cognitive" in nature. Thus, enhanced boundary discrimination may be due to the use of a prototype, but may be a sufficiently late or higher level process to provide little information about the specific, lower level functioning of the prototype.

The categorical perception literature had focused on high discrimination near the category boundary, looking primarily for troughs of chance performance within categories (a trough is a low point between regions of higher levels of performance). The initial goal of Kuhl's research, as well as of the present research, was to examine the perception of stimuli within a single perceptual category. The relevant question for this more modern research should thus be restated: avoiding (possibly higher level) category boundary effects, is within-category performance better or poorer near the category prototype? The stimuli for the present study were all within the category of a $\mathrm{C}$ major chord, and we have found better discrimination near the prototype. The nonprototype stimuli in the Kuhl study may have included at least one category boundary. Thus, the Kuhl NP context discrimination may have reflected the type of cognitive enhancement described above rather than the absence of any special sensory or perceptual attribute of a portion of the stimulus space. This possibility was acknowledged by Iverson and Kuhl (1995).

(Manuscript received January 18, 1994; revision accepted for publication February 13, 1995.) 